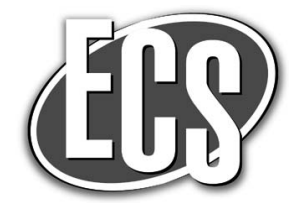

\title{
The Electro-Chemo-Mechanical Coupling in Lithium Alloy Electrodes and Its Origins
}

\author{
Tobias Kitzler, ${ }^{\mathrm{a}, \mathrm{z}}$ Emad Maawad, ${ }^{\mathrm{b}}$ Daniel M. Többens, ${ }^{\mathrm{c}}$ Markus Ziehmer, ${ }^{\mathrm{a}}$ \\ and Jürgen Markmann ${ }^{\mathrm{a}, \mathrm{d}}$
}

${ }^{a}$ Helmholtz-Zentrum Geesthacht, Institute of Materials Research, Materials Mechanics, 21502 Geesthacht, Germany
${ }^{b}$ Helmholtz-Zentrum Geesthacht, Institute of Materials Research, Materials Physics, 21502 Geesthacht, Germany
${ }^{c}$ Helmholtz-Zentrum Berlin for Materials and Energy, Department of Crystallography, 12489 Berlin, Germany
${ }^{d}$ Hamburg University of Technology, Institute of Materials Physics and Technology, 21073 Hamburg, Germany

A method to identify and separate the influence of changes in the surface stress from the bulk stress in a model lithium-ion battery electrode during electrochemical cycling was developed. The strategy for this separation is based on the different influence of surface and bulk stresses on the coupling between electrode potential and mechanical strain as measured by dynamic electro-chemo-mechanical analysis and the coupling between the transferred electric charge and the elastic strain as determined by wide angle X-ray scattering. Using both methods, it was possible to uncover the behavior of an apparent surface stress evoked by the bulk stress due to grain boundary alloying of lithium in a gold film. Additionally, the analysis allowed for a determination of a range in surface stress due to underpotential deposition of one monolayer of lithium as the interval between -3.1 to $-1.9 \mathrm{~N} / \mathrm{m}$.

(C) The Author(s) 2015. Published by ECS. This is an open access article distributed under the terms of the Creative Commons Attribution 4.0 License (CC BY, http://creativecommons.org/licenses/by/4.0/), which permits unrestricted reuse of the work in any medium, provided the original work is properly cited. [DOI: 10.1149/2.0461514jes] All rights reserved.

Manuscript submitted August 17, 2015; revised manuscript received September 21, 2015. Published October 9, 2015.

Lithium-ion batteries are widely used, though the amount of electric charge they can store is still far away from their theoretical capacity. ${ }^{1,2}$ In order to increase the capacity of lithium-ion battery anodes, the commonly used graphite needs to be replaced. A very promising alternative are materials which can form alloys with lithium. ${ }^{3,4}$ There are several possible candidates. ${ }^{5}$ Among the most important ones are silicon, germanium, tin, aluminum, and antimony. The theoretical volumetric lithium storage capacity of these materials is several times higher than that of the currently used anodes. In case of silicon, more than ten times that of graphite relative to the weight of the host material. This storage capability of the afore mentioned materials is based on their ability to store more than one, in case of silicon, germanium, and tin up to 4.4 lithium atoms per host atom. The obvious drawback of these materials is the huge volume change during lithium loading which can easily exceed $100 \%$.

If the electrode material is constrained (e.g. by the current collector) or the lithium is inhomogeneously distributed in the host material, the change in volume is accompanied by stresses. ${ }^{6}$ Possible consequences are the evolution of cracks in the material which can lead to pulverization of the electrode material and consequently loss of capacity. ${ }^{6}$ A promising possibility to prevent pulverization is to reduce the structure size of the electrode material and to introduce high porosity. ${ }^{7,8}$ High porosity reduces concentration gradients by reducing the diffusion lengths and it weakens the constraints by offering free space in the vicinity of the electrode. However, this strategy also increases the surface to volume ratio, and therefore drastically increases the impact of the surface stress, which can have an important impact on the stability of the electrode material. ${ }^{9}$ Additionally, the stress interacts with the chemical potential via the strain energy. ${ }^{10}$ Therefore, the equilibrium lithium concentration is a function of the stress state which, in general, is inhomogeneous within the electrode material. Recent simulations performed by Gau and Zhou ${ }^{11}$ indicated, that the coupling of deformation and diffusion increases the energy release rate and, therefore, promotes crack growth. According to the authors, this coupling is governed by the chemical to mechanical $\tilde{\Omega}^{\mathrm{CM}}$ and mechanical to chemical $\tilde{\Omega}^{\mathrm{MC}}{ }^{11}$ partial atomic volumes. $\tilde{\Omega}^{\mathrm{CM}}$ measures the interplay between the relative increase in volume in a stress-free state at a given lithium concentration, whereas $\tilde{\Omega}^{\mathrm{MC}}$ is a measure for the diffusive migration of lithium content in the host material which depends on local stress and temperature. Those values are typically estimated from equilibrium materials parame- ters (elastic moduli, lattice parameter, and Poisson's ratio) of volume samples.

In this study we measure the potential-strain coupling parameter (PSCP) $\varsigma$ of a thin gold film. The PSCP is a value that can be used to calculate the relative increase of volume per transferred charge $\eta$, in case the bulk modulus and the Poisson's ratio are known. The results indicate, that $\eta$ has a very strong dependency on the electrode potential. Regardless of this potentially crucial impact of the potentialstress coupling, there are only a few cases where the mechanical contribution to the electrode potential was actually measured. ${ }^{12-14}$ To our knowledge no work has been published which actually measured this coupling continuously in a wide potential range.

Only few experiments were performed aiming to measure the electrocapillary effect in lithium electrolytes. ${ }^{15,16}$ Measurements of the change in real surface stress turn out to be rather challenging for lithium alloy electrodes. The standard way for measuring changes of the surface stress (cantilever bending experiments) in such electrodes additionally to surface stress changes measures the change in stress due to alloy formation. This is used e.g. by Sethuraman et al. to measure the stress upon alloy formation. In order to understand the underlying mechanisms it is necessary to separate bulk from surface contributions.

The PSCP is a measure for the change in electrode potential with elastic strain and can be determined by dynamic-electro-chemomechanical analysis (DECMA). ${ }^{17}$ According to thermodynamics the change in electrode potential with elastic strain equals the change in stress with charge density and/or surface stress with surface charge density, depending on the mechanism. DECMA has the advantage, that it can be performed continuously during electrochemical cycling. Since the PSCP is a derivative and the measurement is rather insensitive to slow processes, it cannot be used to measure the absolute changes in surface stress with potential. On the other hand, the PSCP is closely related to the thermodynamics of the system, which makes it a potentially powerful tool for getting a better insight into the processes active on the surface and in the bulk of the electrode.

Since the surface and the bulk of the sample contribute to the PSCP at the same time (which will be shown in Combined surface and bulk PSCP section), additional measurements are required to separate both contributions. The required additional data is obtained by measurements of the lattice spacing by means of in-situ wideangle X-ray scattering. Such a combination of the results requires a model of the electrode. The introduction of such a model finally allows separating surface and bulk contributions to the PSCP. 


\section{Theory}

General response of electrode potential to applied strain.- For surface mechanisms the potential-strain coupling parameter (PSCP) is called the electrocapillary coupling parameter $\left(\mathrm{ECCP} ; \varsigma^{\mathrm{s}}\right)$ and is defined by: ${ }^{18}$

$$
\left.\varsigma^{\mathrm{s}} \equiv \frac{\mathrm{d} f}{\mathrm{~d} q}\right|_{e}=\left.\frac{\mathrm{d} E}{\mathrm{~d} e}\right|_{q}
$$

where $f$ is the surface stress, $q$ is the surface charge density, $E$ is the electrode potential and $e=\epsilon_{11}+\epsilon_{22}\left(e=2 \epsilon_{11}\right.$ in an isotropic case) is the tangential strain at the surface. Equation 1 requires that the surface stress is isotropic in the surface plane. This requirement is always satisfied for an isotropic surface. ${ }^{19}$

The bulk of a thin film electrode can thermodynamically be treated like a surface as long as there is no stresses perpendicular to the surface. In such a case, the stress within the electrode can be described as an apparent surface stress. For the special case of a planar isotropic film, the apparent surface stress, which equals the product of in-plane stress and thickness can be described by a scalar, equivalent to the surface stress. In such a case, the apparent surface stress is related to the in-plane stress by:

$$
f^{\text {app }}=0.5\left(\sigma_{11}+\sigma_{22}\right) h:=\sigma_{\|} h
$$

where $f^{\text {app }}$ is the apparent surface stress, $\sigma_{11}$ and $\sigma_{22}$ are the 11 and 22 components of the Cauchy stress tensor, $\sigma_{\|}$is the scalar stress parallel to the film and $h$ is the thickness of the film. The PSCP of the bulk $\varsigma^{\mathrm{b}}$ can in this case be described similarly to the PSCP of the surface by:

$$
\left.\varsigma^{\mathrm{b}} \equiv \frac{\mathrm{d} f^{\text {app }}}{\mathrm{d} q^{\text {app }}}\right|_{e, \sigma_{\perp}}=\left.\frac{h \mathrm{~d} \sigma}{\mathrm{d} q_{\text {app }}}\right|_{e, \sigma_{\perp}}=\left.\frac{\mathrm{d} E}{\mathrm{~d} e}\right|_{q^{\text {app }, \sigma_{\perp}}}
$$

where $q^{\text {app }}$ is the apparent surface charge density, which measures the charge transfer across the electrode electrolyte interface correlated to changes in the bulk lithium concentration, $e$ is the tangential strain of the film and $\sigma_{\perp}$ is the stress perpendicular to the surface.

Combined surface and bulk PSCP.- By DECMA, the surface stress and the apparent surface stress cannot be distinguished. The same is true for the surface charge density and the apparent surface charge density. As a consequence, the PSCP is sensitive to surface and bulk contributions according to:

$$
\left.\frac{\mathrm{d} E}{\mathrm{~d} e}\right|_{q+q^{\text {app }}}=\left.\frac{\mathrm{d}\left(f+f^{\text {app }}\right)}{\mathrm{d}\left(q+q^{\text {app }}\right)}\right|_{e}
$$

Using $f=f(q)$ and $f^{\text {app }}=f^{\text {app }}\left(q^{\text {app }}\right)$ leads to:

$$
\left.\frac{\mathrm{d} f^{\text {app }}}{\mathrm{d} q^{\text {app }}} \frac{\mathrm{d} q^{\text {app }}}{\mathrm{d}\left(q+q^{\text {app }}\right)}\right|_{e}+\left.\frac{\mathrm{d} f}{\mathrm{~d} q} \frac{\mathrm{d} q}{\mathrm{~d}\left(q+q^{\text {app }}\right)}\right|_{e}
$$

Since the surface and the bulk need to be at the same electrical potential, the derivatives of the different charges with respect to the total charge can be replaced by the share of the surface $\left(C_{\mathrm{s}} /\left(C_{\mathrm{s}}+C_{\mathrm{b}}\right)\right)$ and the bulk $\left(C_{\mathrm{b}} /\left(C_{\mathrm{s}}+C_{\mathrm{b}}\right)\right)$ on the overall capacity. In case of nonequilibrium conditions, $C_{\mathrm{b}}$ and $C_{\mathrm{s}}$ have to be considered as effective capacities of the bulk and the surface. In conclusion, Equation 4 can be divided into :

$$
\left.\frac{\mathrm{d} E}{\mathrm{~d} e}\right|_{q+q^{\mathrm{app}}}=\left(\left.C_{\mathrm{s}} \frac{\mathrm{d} E}{\mathrm{~d} e}\right|_{q}+\left.C_{\mathrm{b}} \frac{\mathrm{d} E}{\mathrm{~d} e}\right|_{q^{\mathrm{app}, \sigma_{\perp}}}\right) \frac{1}{C_{\mathrm{s}}+C_{\mathrm{b}}}
$$

After introduction of the corresponding PSCPs, this can be written as:

$$
\left.\frac{\mathrm{d} E}{\mathrm{~d} e}\right|_{q+q^{\mathrm{app}}}=\left(C_{\mathrm{s}} \varsigma^{\mathrm{s}}+C_{\mathrm{b}} \varsigma^{\mathrm{b}}\right) \frac{1}{C_{\mathrm{s}}+C_{\mathrm{b}}} \equiv \varsigma^{\mathrm{c}}
$$

The combined PSCP $\varsigma^{\mathrm{c}}$ is an effective capacity weighted average of the reaction of the surface and the bulk to strain. In the following, the total, i.e. combined, PSCP is directly measured and the bulk and surface contributions are separated by analysis of the changes of the lattice spacing. The corresponding PSCP of the surface is then derived by:

$$
\varsigma^{\mathrm{s}}=\frac{\varsigma^{\mathrm{c}}\left(C_{\mathrm{s}}+C_{\mathrm{b}}\right)-C_{\mathrm{b}} \varsigma^{\mathrm{b}}}{C_{\mathrm{s}}}
$$

It can be expected, that the dynamics of the surface is higher than the dynamics of the bulk. Hence, the surface contribution to the combined PSCP will be more dominant when $\varsigma^{\mathrm{c}}$ is measured by fast strain variations.

Interpretation of the PSCP. - The PSCP of the surface couples the changes in surface stress to the surface charge density. Hence, the integration of $\varsigma^{\mathrm{s}} \mathrm{d} q$ equals the changes in surface stress. The origin of this changing surface stress can be diverse. Well known possibilities are charging of the surface, adatom adsorption, underpotential deposition $^{18}$ (deposition of roughly one monolayer at an electrode potential higher than the required potential for bulk deposition) and the formation of a surface alloy (alloy formation on the topmost atomic layer(s) upon deposition of one metal onto another).

The bulk behaves slightly different. For all relevant phenomena discussed in this work the bulk of a metallic electrode can be assumed to be at a constant electric potential and to carry no net charge. This means mechanisms like charging can be excluded as the source of the PSCP of the bulk leaving only changes of the lattice parameter and creation of additional lattice places as possible underlying processes.

For adsorption of hydrogen in a thin superficial layer of nanoporous palladium and absorption of hydrogen in the bulk of nanoporous palladium, where hydrogen occupies interstitial lattice places, the PSCP (also called the apparent electrocapillary coupling parameter) was calculated and measured by Viswanath and Weissmüller. ${ }^{20}$ Two of the main results of their study can be summarized by:

$$
\begin{gathered}
\mathrm{S}_{\mathrm{ab}}^{\mathrm{app}}=\frac{9 K \widehat{\eta} \Omega}{2 z q_{0}} \\
\mathrm{~S}_{\mathrm{ad}}=\frac{3 K \widehat{\eta} \Omega}{z q_{0}} \frac{1-2 \nu}{1-\nu}
\end{gathered}
$$

where $\varsigma_{\mathrm{ab}}^{\mathrm{app}}$ is the PSCP for bulk absorption, which implies that it is a measure for the change in the apparent surface stress with apparent surface charge density in case all strain components are constant. $\zeta_{\text {ad }}$ is the ECCP for hydrogen adsorption on a superficial layer, which implies that it is a measure for the changes in surface stress with surface charge density where the strain parallel to the surface and the stress normal to the surface are constant. $K$ is the bulk modulus of the initial material, $\Omega$ is the atomic volume of the ab- or adsorbed species (below "active" species), $q_{0}$ is the elementary charge, $v$ is the Poisson's ratio of the host material and $\widehat{\eta}$ is the change in lattice parameter with active material concentration. The original formulation ${ }^{20}$ does not contain $z$ because it was set to the valency of hydrogen which is $1 . \widehat{\eta}$ is defined by the equation: $a=a_{0}\left(1+\widehat{\eta} N_{\text {active }} / N_{\text {host }}\right)$, where $a$ is the lattice parameter of the material, $a_{0}$ is the lattice parameter for $N_{\text {active }}=0$, $N_{\text {active }}$ is the number of atoms of the active material and $N_{\text {host }}$ is the number of atoms of the host material and therefore a constant.

In contrast to interstitial solid solutions, there is no direct correlation of the stress-free strain to the lattice parameter. This prevents the usage of Equations 9 and 10 for substitutional solid solutions like the gold-lithium system under investigation, which forms a substitutional solid solution for low lithium concentrations..$^{21}$ Nevertheless, both equations can be adapted for a substitutional alloy. In order to do so $\widehat{\eta}$, needs to be replaced by a more general property $\eta$, that is not directly linked to the lattice parameter but numerically equals $\widehat{\eta}$ for interstitial solid solutions. This is satisfied for $\eta$ defined by :

$$
\eta=\left.\frac{N_{\text {host }}}{2} \frac{\mathrm{d} e^{\text {sf }}}{\mathrm{d} N_{\text {active }}}\right|_{\sigma_{i j}}
$$

where $e^{\mathrm{sf}}$ is the tangential, stress-free strain with respect to the unalloyed metal, $N_{\text {active }}$ is the number of atoms of the active material, $N_{\text {host }}$ is the number of atoms of the host material and therefore a constant. 
Interrelation of real and apparent surface stress changes with the lattice parameter- - The interrelation of surface stress and lattice parameter differs from the interrelation of the apparent surface stress and the lattice parameter. This is due to the fact that a surface stress acts on the sample and the substrate in a similar matter, whereas a bulk stress (apparent surface stress) quantifies the force of the sample on the substrate. Hence, the strain in the sample needs to be anti-parallel to the strain in the substrate.

In order to quantify the interrelation, a model of the electrode is required. A first estimation uses a layered sample, which corresponds to the experimental samples, with homogeneous in-plane strain in all layers. If equality of forces is required, a surface stress leads to:

$$
\begin{gathered}
f+\left(B_{\mathrm{Pi}} h_{\mathrm{Pi}}+B_{\mathrm{Ti}} h_{\mathrm{Ti}}+B_{\mathrm{Au}} h_{\mathrm{Au}}\right) \epsilon_{\|}^{\mathrm{e}}=0 \\
\Rightarrow \epsilon_{\|}^{\mathrm{e}}=\frac{-f}{B_{\mathrm{Pi}} h_{\mathrm{Pi}}+B_{\mathrm{Ti}} h_{\mathrm{Ti}}+B_{\mathrm{Au}} h_{\mathrm{Au}}}
\end{gathered}
$$

where the $B_{\mathrm{Pi}}, B_{\mathrm{Ti}}, B_{\mathrm{Au}}$ are the biaxial moduli of polyimide, titanium, and gold, $h_{\mathrm{Pi}}, h_{\mathrm{Ti}}, h_{\mathrm{Au}}$ are the corresponding thicknesses and $\epsilon_{\|}^{\mathrm{e}}$ is the elastic in-plane strain of the sample.

In case of an apparent surface stress the situation is different because the total in-plane strain of the sample material $\epsilon_{\|}^{\mathrm{t}}$ is the sum of the elastic $\epsilon_{\|}^{\mathrm{e}}$ and the stress-free in-plane strain $\epsilon_{\|}^{\text {sf }}$ of the sample.

$$
\epsilon_{\|}^{\mathrm{t}}=\frac{-f_{\text {app }}}{B_{\mathrm{Pi}} h_{\mathrm{Pi}}+B_{\mathrm{Ti}} h_{\mathrm{Ti}}}
$$

Using Equation 2, this leads to:

$$
\epsilon_{\|}^{\mathrm{e}}=-\epsilon_{\|}^{\mathrm{sf}} \frac{B_{\mathrm{Pi}} h_{\mathrm{Pi}}+B_{\mathrm{Ti}} h_{\mathrm{Ti}}}{B_{\mathrm{Pi}} h_{\mathrm{Pi}}+B_{\mathrm{Ti}} h_{\mathrm{Ti}}+B_{\mathrm{Au}} h_{\mathrm{Au}}}
$$

The elastic strain is always smaller than the stress-free strain and its direction anti-parallel to it. Therefore the elastic strain is always anti-parallel to the total strain as well.

\section{Experimental}

Sample preparation.- The samples were prepared by DC magnetron sputtering on polyimide foil ( $75 \mu \mathrm{m}$ thick, $5 \mathrm{~cm}$ in diameter) for $\mathrm{X}$-ray diffraction experiments and on polyimide based resistive strain gage $\left(H B M\right.$ 1-LY11-10/120; $18 \times 9.5 \times 0.1 \mathrm{~mm}^{3}$ ) for DECMA, where the sample material occupies a circular area with a diameter of $8 \mathrm{~mm}$. Prior to deposition the substrates were cleaned by argon plasma etching and transferred to the sputter chamber without contact to air. Base pressure and working pressure in the sputter chamber were $<4 \cdot 10^{-8} \mathrm{mbar}$ and $5.7 \cdot 10^{-3}$ mbar respectively. The working pressure was established by a constant flow of 7 SCCM (standard cubic centimeter per minute) argon 5.0. All layers were sputtered using power regulation mode. Sample target distance was $\approx 15 \mathrm{~cm}$ for gold and titanium and $\approx 7 \mathrm{~cm}$ for copper. Titanium, gold and copper (all 99.99\% purity) were sputtered at $100(\mathrm{Ti})$ and $50 \mathrm{~W}(\mathrm{Au}, \mathrm{Cu})$ constant power. The thickness was controlled by sputtering time. For samples with defined shape (DECMA), a stainless steel shadow mask was used. After preparation, the samples were stored in a laboratory type drying chamber at $125^{\circ} \mathrm{C}$ for several days for homogenization and at $90^{\circ} \mathrm{C}$ at a heating plate inside the glove box for several hours to remove water adsorbate.

The sample for DECMA consists of $\approx 20 \mathrm{~nm}$ Ti as adhesion layer and $\approx 55 \mathrm{~nm} \mathrm{Au}$ on a polyimide based resistive strain gage with defined shape. The sample for in-situ X-ray diffraction was $\approx 20 \mathrm{~nm}$ $\mathrm{Ti}$ as adhesion layer and $\approx 55 \mathrm{~nm} \mathrm{Au}$ on a polyimide foil with $\approx 150 \mathrm{~nm}$ $\mathrm{Cu}$ on the opposite side.

Dynamic electro-chemo-mechanical analysis (DECMA).- The design of the DECMA stage was inspired by the one used in Ref. 17. The changes were mainly focusing on the possibility for operation inside a glove box. Further modifications involved increased stiffness, higher accessible frequencies and faster as well as more precise strain measurement. For operation inside a glove box, it was sufficient to a)

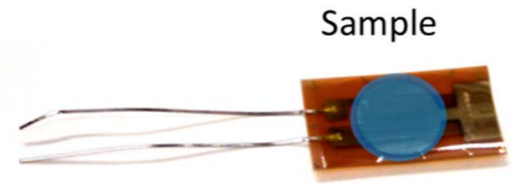

b)

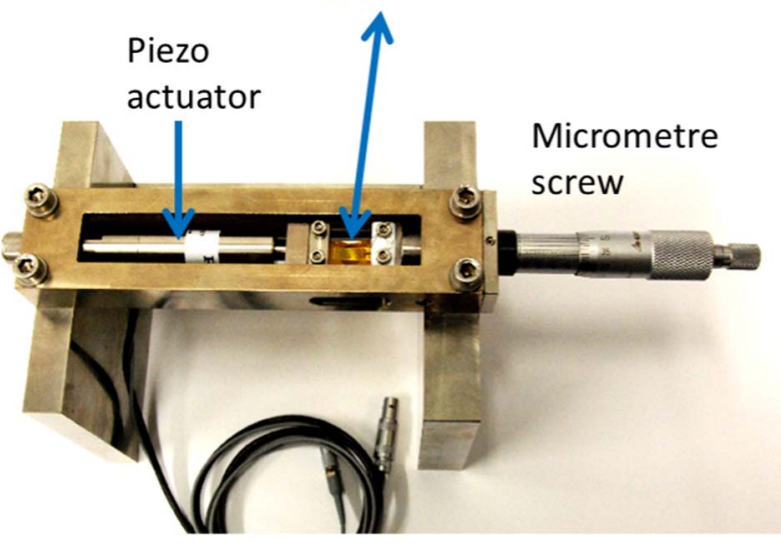

Figure 1. a) Picture of a sample with the contact area of the electrolyte colored in blue. b) Picture of the DECMA-stage mounted on a rack.

omit the housing and to provide the electrical connections. The stiffness increase was achieved by using a massive titanium frame which connects the piezo actuator and the micrometer screw. A clamp is attached to each of these two parts for mounting the samples. The frequency range could be expanded by using a smaller and therefore faster piezo actuator. An improved strain measurement was achieved by using polyimide based resistive strain gages (HBM 1-LY11-10/120; $\left.18.0 \times 9.5 \times 0.1 \mathrm{~mm}^{3}\right)$ as substrates and a measurement amplifier (HBM MX410 highly dynamic universal amplifier) to dynamically evaluate the strain. A picture of this device and a sample are shown in Figure 1.

By using a very small amount of electrolyte the total amount of impurities and the possibility for dissolving components of the solid-electrolyte interface, ${ }^{22-24}$ which leads to additional electrolyte decomposition and therefore Faraday currents, was kept small. This was achieved by using a stainless steel rod with a small lithium containment on top, which was filled with lithium of a purity of $99.9 \%$. By adding some drops of electrolyte (roughly 50-100 $\mu 1$ ) a standing meniscus was formed and used to contact the sample. The electrolyte was LP 30 from BASF ( $1 \mathrm{~mol} \mathrm{LiPF}_{6}$ in a 1:1 mixture of ethylene carbonate and dimethyl carbonate; the purities were 99.80, 99.95 and $99.98 \%$ respectively). In this way, a two electrode electrochemical cell was created. An additional advantage of this two electrode electrochemical cell was the noise reduction of the DECMA signal compared to the three electrode electrochemical cell used for test measurements, without showing any noticeable disadvantages.

The sample material was sputtered on the smooth side of the resistive strain gage. After the sample was clamped between the piezo actuator and the micrometer screw, it was pre-strained to roughly $0.5 \%$. This was necessary to allow the piezo actuator to apply periodic variations of the strain. Both, the amplitude of the variation and the applied pre-straining were calculated from the change in resistance of the resistive strain gage. The amplitude of the strain variation was measured twice, one time in the beginning of the experiment and once after it ended. The value changed less than $1 \%$. In order to evaluate the phase shift between electrical and mechanical signal, the analog strain signal of the measurement amplifier was used as a reference signal for a lock-in amplifier (SIGNAL RECOVERY LIA 7265), which was used for measuring the potential variations due to strain variation. This lock-in amplifier was operated in A-B mode with the sample (working electrode) connected to input $\mathrm{A}$ and the counter electrode to input $\mathrm{B}$. The sensitivity was set to $1 \mathrm{mV}$. For measuring during cyclic voltammetry $(\mathrm{CV})$ working and counter electrode were connected to 


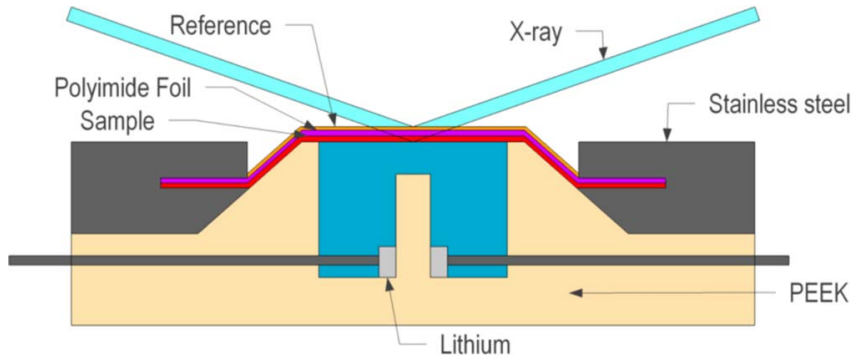

Figure 2. Sketch of the in-situ electrochemical cell. PEEK stands for polyether ether ketone.

the potentiostat. In order to prevent the potentiostat from counteracting the strain induced potential variation, the working electrode was decoupled from the potentiostat by a $50 \mathrm{k} \Omega$ resistor. Together with the capacitance of the sample the resistor created a low-pass filter. ${ }^{25}$ For having comparable $\mathrm{CV}$ data, the influence of the resistor on the electrode potential had to be recalculated. This was done by using:

$$
U_{\text {sample }}=U_{\text {potentiostat }}-R * I
$$

Due to this current dependent impact of the resistor on the potential, the scan speed $(U)$ at the sample is not constant anymore. Deviation of the measured current from a current one would expect at constant scan speed was estimated by assuming purely capacitive currents to be less than $10 \%$. This impact can shift features on the potential axis for $\approx 50 \mathrm{mV}$

The measured potential variations due to the variation of strain are used to calculate the PSCP

$$
\varsigma \approx \varsigma_{\text {re }}=\frac{\operatorname{Re}\left(U_{\mathrm{ac}}\right)}{\left(1-\nu_{\text {substrate }}\right) \epsilon_{\text {substrate }}}
$$

where $\operatorname{Re}\left(U_{\mathrm{ac}}\right)$ is the root mean square (RMS) of the potential variation that is in phase with the strain variation, $v_{\text {substrate }}$ is the Poisson's ratio of the substrate, which according to Ref. 26 is 0.34 at $1 \%$ of uniaxial strain, and $\epsilon_{\text {substrate }}$ is the RMS of the uni-axial substrate strain variation. The phase shift of the signal is typically associated with finite transport rates in the electrolyte or slow adsorption or absorption rates ${ }^{17}$ and will therefore be neglected.

In-situ measurements of the lattice spacing.- A customized insitu electrochemical cell (Figures 2 and 3) was used for in-situ X-ray diffraction. It was designed for measuring $\mathrm{X}$-ray diffraction patterns even at low incidence angles in totally arbitrary sample orientations. The sample is clamped between two stainless steel rings. These rings were then attached to the body (made from PEEK) of the electrochem-

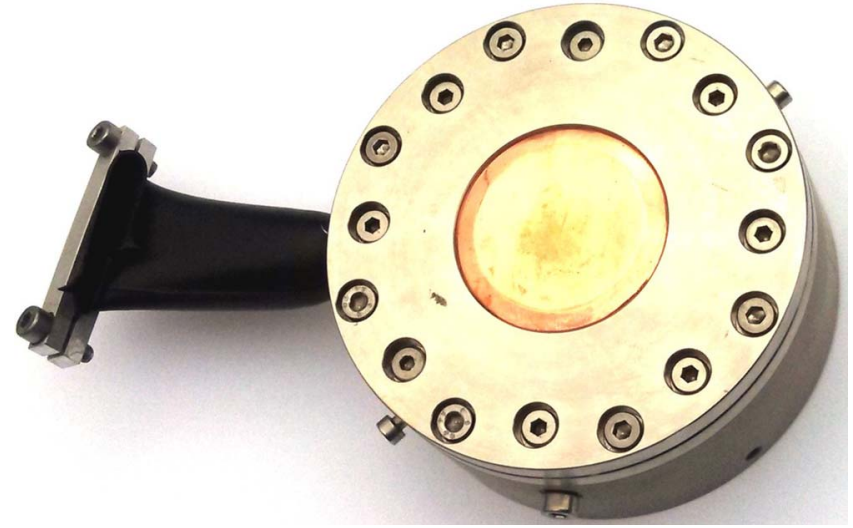

Figure 3. Picture of the in-situ electrochemical cell. In the center of the cell is the copper-gold colored sample. Around the sample the stainless steel rings are visible. The black part on the left hand side is the butyl bladder. ical cell. The body is designed in such a way that it pushes the sample, that acts as the X-ray window at the same time, to the highest point of the cell. During this attachment, the sample is slightly strained and thereby fixed. The electrode-electrolyte interface in this electrochemical cell is $7.1 \pm 0.5 \mathrm{~cm}^{2}$. The electrolyte used was as in the DECMA setup LP 30 as purchased. Counter and reference electrodes were 99.9\% Li foil contacted by stainless steel screws. For compensation of pressure changes a small butyl bladder was attached to the cell. All parts of the electrochemical cell were stored for at least 7 days in a laboratory type drying chamber at $100^{\circ} \mathrm{C}$. In case it was necessary to open the electrochemical cell between measurements in the open air for cleaning, it was cleaned without using water and dried using a hot air fan for at least 30 minutes.

The measurements were performed by using in-situ X-ray diffraction at the KMC- 2 bending magnet beamline ${ }^{27}$ of the synchrotron facility BESSY-II (HZB, Berlin, Germany) using a radiation wavelength of $1.5406 \AA$. The endstation diffraction at KMC- 2 is equipped with a Huber 6-circle goniometer in psi-geometry and a 2-dimensional micro gap detector with a spatial resolution of $70 \mu \mathrm{m}$ (VANTEC 2000). Measurements were done in reflection geometry, using the $\chi$-circle to tilt the sample surface between in-plane and symmetric out-of-plane orientation. X-ray absorption in the polyimide substrate limited the in-plane geometry to a minimal tilt angle of $\chi=10^{\circ}$. Careful centering of all goniometer axes and the sample surface against the primary beam ensured the absence of any significant displacement errors. Raw 2D-data were converted into intensity vs. scattering angle line profiles by integration along Debye-cones. Based on these data peak positions and full widths at half maximum (FWHM) were determined by fitting of pseudo-Voigt functions. ${ }^{28}$

\section{Results}

Dynamic electro-chemo-mechanical analysis.-DECMA was performed simultaneously with cyclic voltammetry. In order to highlight the history-dependence of the result, the potential window was increased successively starting with $[2 \mathrm{~V}$ to $1 \mathrm{~V}]$ and decreasing the minimum potential after every 3 cycles to $0.7,0.5,0.4,0.3,0.25 \mathrm{~V}$. The maximum potential was kept at $2 \mathrm{~V}$. The results for the $55 \mathrm{~nm}$ thick gold film are shown in Figure 4.

This measurement allows correlating the features of the cyclic voltammogram to the features of the PSCP. The most distinct feature in the cyclic voltammogram is the peak at roughly $1 \mathrm{~V}$ vs. $\mathrm{Li} / \mathrm{Li}^{+}$. Literature results link this peak to the stripping of the lithium underpotential deposit on a gold surface. ${ }^{29}$

In the potential range of $2 \mathrm{~V}$ to $1 \mathrm{~V}$ all curves show a very similar, almost linear behavior (see Figure 4). For lower potentials a hysteresis appears. This hysteresis is more pronounced for cycles which reach lower potentials and it diminishes between 0.9 and $1.2 \mathrm{~V} \mathrm{vs.} \mathrm{Li} / \mathrm{Li}^{+}$. In this range, a plateau is visible in the positive scan direction. This plateau appears at the same time as the underpotential deposition stripping (UPDs) peak becomes visible in the CV curve.

In-situ measurements of the lattice spacing. - On the opposite side of the samples for in-situ X-ray diffraction a roughly $150 \mathrm{~nm}$ thick copper film was sputtered which was used as a reference. The sample was strained when it was mounted, therefore only changes in the strain values relative to an arbitrary reference state are used. This reference state was chosen to be the second value in each plot, because the first one showed a slightly bigger scatter due to the starting of the electrochemical cycling.

The measurements were as during DECMA performed during cyclic voltammetry starting with a potential window of $2 \mathrm{~V}$ to $1 \mathrm{~V}$ and reducing the lower vertex potential to $0.7,0.5,0.4,0.3,0.25 \mathrm{~V}$ after every two cycles. During the first of those cycles diffraction patterns were recorded at a tilting angle of $\chi=90^{\circ}$ to measure the out-ofplane lattice parameter. During the second cycle, diffraction patterns were recorded at a tilting angle of $\chi=10^{\circ}$ to measure the in-plane lattice parameter. In both cases the incident angle $\Theta$ was $20.5^{\circ}$ and the 


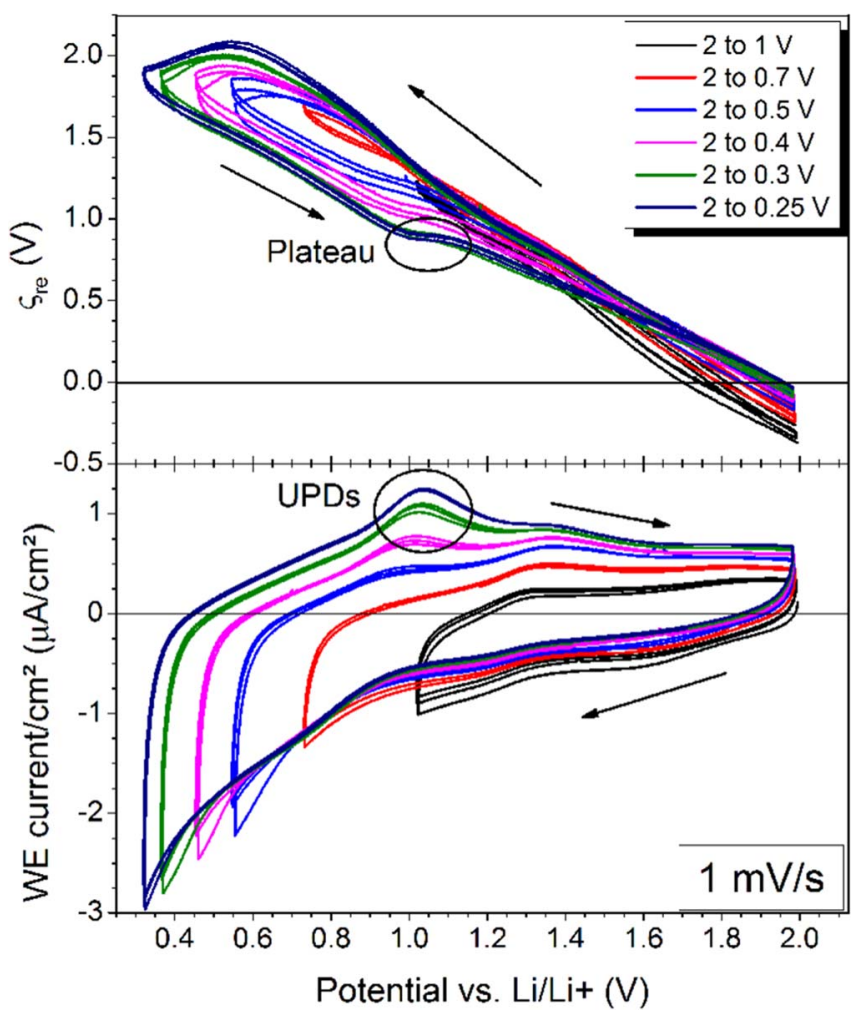

Figure 4. Potential strain coupling parameter (top) and cyclic voltammetry (bottom) results for a $55 \mathrm{~nm}$ thick gold film during cyclic voltammetry for different potential ranges (indicated by colors), measured at a scan speed of $1 \mathrm{mV} / \mathrm{s}$ with a straining frequency of $20 \mathrm{~Hz}$ and a strain variation of $110 \mu \mathrm{m} / \mathrm{m}$ RMS.

detector recorded intensities for reflection angles $2 \Theta$ in the range from $35.7^{\circ}$ to $45.9^{\circ}$. The recorded patterns included the gold and copper (111)-peaks.

Before every measurement cycle the potential was slowly reduced to $2 \mathrm{~V}$. During cyclic voltammetry every $50 \mathrm{~s}$ one reflection pattern was recorded with an acquisition time of $20 \mathrm{~s}$. Between two different $\mathrm{CV}$ cycles the potential was kept at $2 \mathrm{~V}$. The peak positions of the gold and copper (111)-peaks are displayed in Figure 5. They were evaluated by fitting the diffraction patterns recorded at a tilting angle of $\chi=10^{\circ}$ to pseudo Voigt functions. The lattice parameter of the copper film only shows a small and monotonous drift. In contrast to that, the lattice

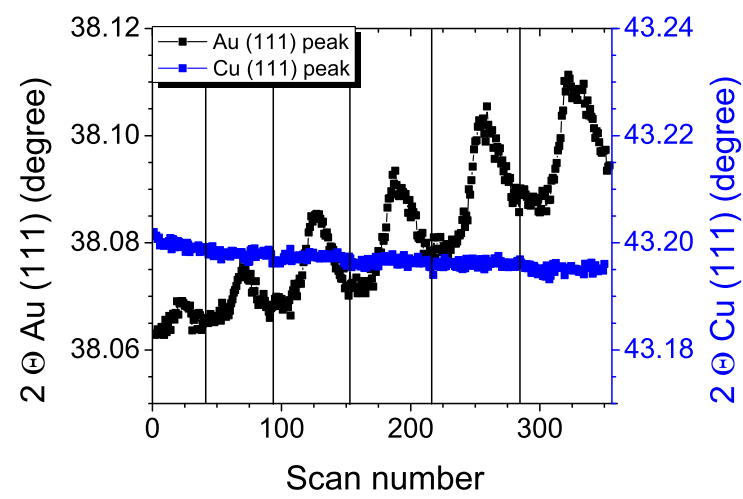

Figure 5. (111)-peak-position vs. scan number during $6 \mathrm{CV}$ cycles. The results of the gold film (black) and the copper reference film (blue) were measured simultaneously at a tilting angle of $\chi=10^{\circ}$. The vertical lines separate the different CVs and thereby indicate the time gaps during which the same measurement for a tilting angle of $\chi=90^{\circ}$ were performed.

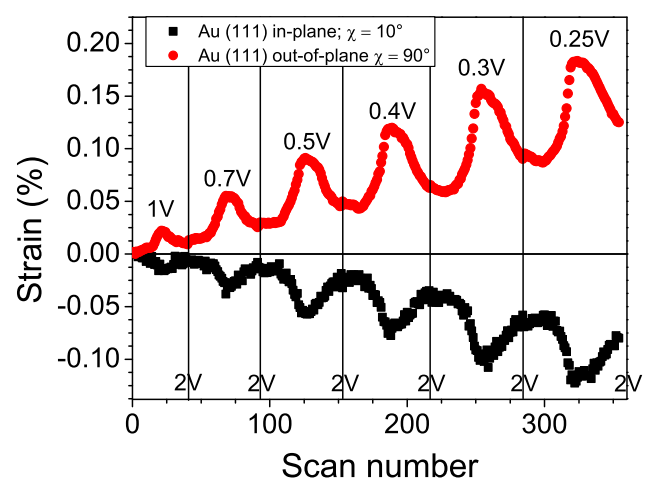

Figure 6. Relative changes of the in-plane (black) and out-of-plane (red) lattice parameter during cyclic voltammetry. The value of scan number two was chosen as the reference state and is therefore zero. The periodic variations as well as the drift have an inverse sign depending on the tilting angle. The vertical black lines separate the cyclic voltammograms and indicate the time-gap during which the measurement at the other tilting angle was performed.

parameter changes of the gold film are much more pronounced, mainly reversible with electrochemical cycling, and show a much higher drift in inverse direction. Diffraction patterns recorded at a tilting angle of $\chi=90^{\circ}$ show a similar, even stronger behavior, but the changes in the gold lattice parameter are in the opposite direction. Figure 6 shows the change of the strain values relative to the second measurement during $6 \mathrm{CV}$ cycles. They were calculated form peak positions of the gold (111)-peak measured at a tilting angle of $\chi=90^{\circ}$ and $\chi=10^{\circ}$. Both, in-plane as well as out-of-plane strain changes are mainly reversible with cycling but occur in inverse directions. As before, scan number two was chosen as the reference and the corresponding strain value is therefore set to zero. Figure $7 \mathrm{~b}$ shows the linear correlation of in-plane to out-of-plane strain changes. The determined proportionality factor is -0.61 .

\section{Discussion}

Dynamic electro-chemo-mechanical analysis.-Figure 4 shows the appearance of a hysteresis in the potential-strain coupling parameter for scans with wide potential windows. The width of the hysteresis mainly increases in the potential range from 1.2 to $0.9 \mathrm{~V} \mathrm{vs.} \mathrm{Li}^{2} / \mathrm{Li}^{+}$. In this potential range the underpotential deposition (UPD) peak in the positive scan direction of the cyclic voltammogram appears. For scans without hysteresis, no underpotential deposition stripping peak is visible. These two features indicate, that the underpotential deposition is responsible for the observed hysteresis.

At the maximum of the underpotential deposition peak, the PSCP hardly changes with potential. Using Equation 8 and estimating, that the effective capacities are proportional to the currents measured during cyclic voltammetry, this result allows calculating an upper and a lower bound for the surface stress induced by one monolayer of lithium on the surface of the sample. At the maximum of the underpotential deposition stripping peak the combined PSCP was measured to be $0.9 \mathrm{~V}$. Since the PSCP measured at the same potential but without underpotential deposit is higher $(1.35 \mathrm{~V}$; as measured during the scan from $2 \mathrm{~V}$ to $700 \mathrm{mV}$ ), the potential-strain coupling parameter of the surface needs to be lower than $0.9 \mathrm{~V}$.

The lower bound for the PSCP of lithium UPD can be calculated by estimating the capacitance of both processes involved. For this purpose we compare the currents measured during cyclic voltammetry of both scan directions $\left(0.50 \mu \mathrm{A} / \mathrm{cm}^{2}\right.$ and $1.23 \mu \mathrm{A} / \mathrm{cm}^{2}$ for the negative and positive scan direction respectively $\Rightarrow C_{\mathrm{s}}: C_{\mathrm{c}}=0.73: 1.23$ and $C_{\mathrm{b}}$ : $C_{\mathrm{c}}=0.5: 1.23$ ). This estimation will give a lower bound, because the dynamics of the surface is typically faster than the dynamics of the bulk. As a consequence, the effective capacitance of the bulk will decline more with increasing frequency than that of the surface. Using again Equation 8 with $\varsigma^{\mathrm{b}}=1.35 \mathrm{~V}$ and $\varsigma^{\mathrm{c}}=0.9 \mathrm{~V}$, these estimations 


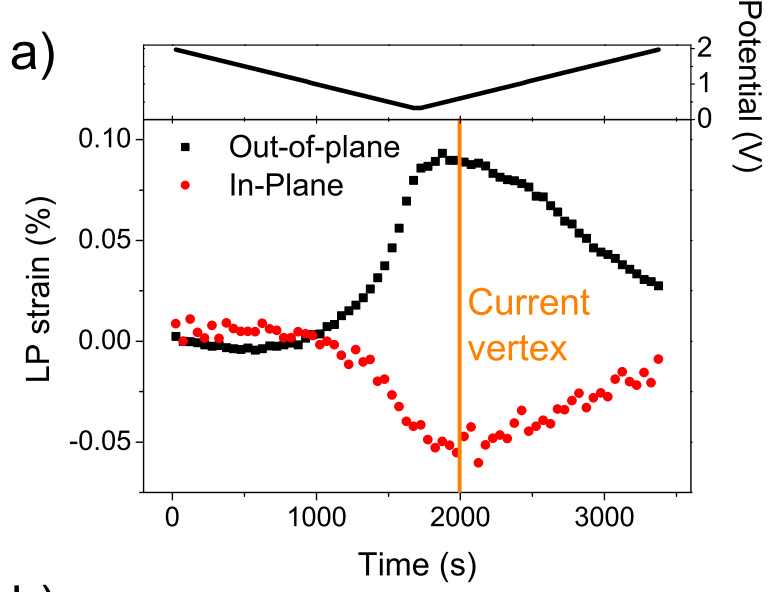

b)

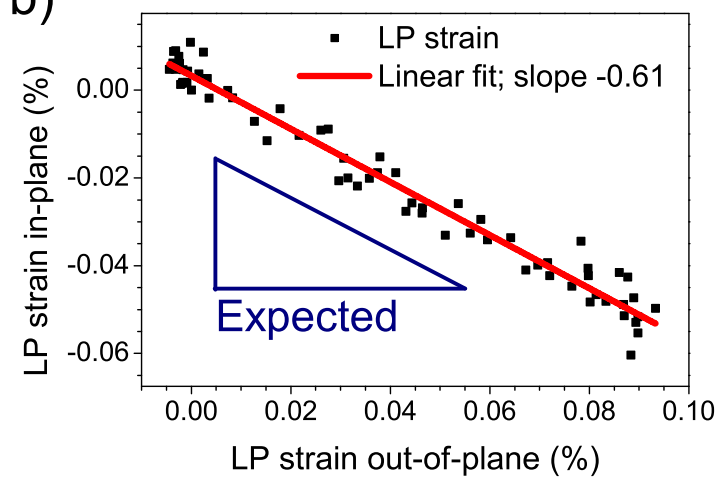

Figure 7. a) Relative changes of the (111)-lattice parameter measured during $\mathrm{CV}$ from $2 \mathrm{~V}$ to $300 \mathrm{mV}$ for tilting angle $\chi=90^{\circ}$ (black) and $\chi=10^{\circ}$ (red) of a $55 \mathrm{~nm}$ Au sample. The orange line indicates the current vertex from cyclic voltammetry data. The applied potential is displayed directly above. b) The tilting angle dependent changes of the lattice parameter plotted against each other in comparison to the slope expected for a planar stress state $(-0.58)$ is indicated.

lead to a lower bound of $0.58 \mathrm{~V}$ for the PSCP of lithium UPD. In order to calculate the changes in surface stress, we additionally need a surface charge density $\Delta q$ corresponding to the deposition of one monolayer of lithium. Due to the (111)-texture of the sample (very similar to the samples used by Smetanin et al. ${ }^{30}$ ), the surface charge density can be calculated by:

$$
\Delta q=-q_{0} \frac{2}{\sqrt{3} d_{111}^{2}}
$$

where $d_{111}$ is the lattice spacing of gold (111) planes $(2.35 \AA)$. This charge density $\left(3.35 \mathrm{C} / \mathrm{m}^{2}\right)$ together with the upper and lower bound of the PSCP of underpotential deposition leads to $-3.0 \mathrm{~N} / \mathrm{m}<\Delta f_{\mathrm{UPD}}<$ $-1.9 \mathrm{~N} / \mathrm{m}$ for the deposition of one monolayer of lithium on the sample surface. Recently Tavassol et al. ${ }^{16}$ published density functional theory results of $\Delta f_{\mathrm{UPD}}$. Their result was $-2.8 \mathrm{~N} / \mathrm{m}$ which lies well within our interval.

In-situ measurements of the lattice spacing. - The periodic variation of the lattice parameter of the gold film upon electrochemical cycling is shown in Figure 5. The lack of periodic variations of the lattice parameter of the copper reference clearly shows the electrochemical nature of these variations. The dependency of this periodic variation on the tilting angle, as shown in Figure 7a, indicates a planar stress state. The in-plane to out-of-plane lattice parameter variation ratio can be calculated using linear elasticity to be $(v-1)$ : 1 . For gold, which has a Poisson's ratio of $0.42^{31}$ in-plane to out-of-plane variations should show a relation of $(-0.58: 1)$ for a planar stress state, which is very close to the measured ratio of $(-0.61: 1)$ as shown

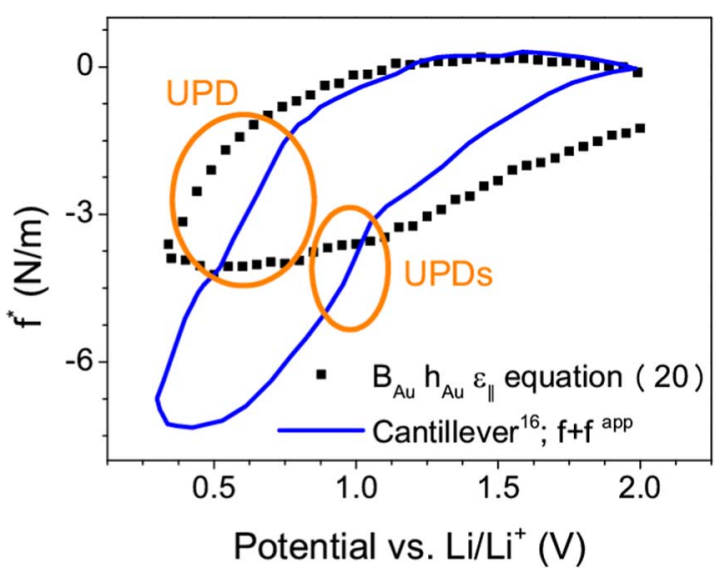

Figure 8. Stress thickness product calculated from X-ray data in comparison to cantilever bending results extracted from. ${ }^{16}$ Except for UPD and UPDs both curves show a similar behavior.

in Figure 7b. Similarly to the periodic variations, the drift shows a strong dependency on the tilting angle. Hence the origin of this drift can be expected to be an irreversible planar stress state. We have identified two possible "measurement artifacts" which could be the source for this drift. The first one is a residual stress due to plastic deformation of the titanium adhesion layer and the second one is an irreversible change of the surface stress due to SEI formation. Both possible artifacts require a deformation of the substrate. Hence the drift is an indication for a soft substrate. Besides the indication of a soft substrate, no further conclusions will be drawn from the drift.

A planar stress state can be a result of a surface stress or a measure for an apparent surface stress. As calculated in Interrelation of real and apparent surface stress changes with the lattice parameter section, surface stress and apparent surface stress have an inverse impact (in direction, not in magnitude) on the lattice parameter. On the other hand, surface stress and apparent surface stress changes have a similar impact on the PSCP and on cantilever bending results. Cantilever bending measures the stress on the substrate and therefore:

$$
f\left(\epsilon^{\mathrm{t}}=0\right)+f^{\text {app }}\left(\epsilon^{t}=0\right)=f_{\text {cantilever }}^{\text {app }}
$$

Elastic changes of the lattice parameter on the other hand are influenced by the real and apparent surface stress according to:

$$
\epsilon_{\|}^{\mathrm{e}} B_{\mathrm{Au}} h_{\mathrm{Au}}=f^{\mathrm{app}}\left(\epsilon^{\mathrm{t}}\right)-\frac{f\left(\epsilon^{\mathrm{t}}\right) B_{\mathrm{Au}} h_{\mathrm{Au}}}{B_{\mathrm{Pi}} h_{\mathrm{Pi}}+B_{\mathrm{Ti}} h_{\mathrm{Ti}}+B_{\mathrm{Au}} h_{\mathrm{Au}}}
$$

This difference can be used to separate both contributions. A qualitative comparison is shown in Figure 8. The comparison shows a good correlation of the apparent surface stress from bulk mechanisms and cantilever bending results. Qualitative differences between apparent surface stress and cantilever bending results are mainly visible where underpotential deposition and stripping occurs. This allows the conclusion, that the major contributions to the electro-chemo-mechanical coupling are underpotential deposition and bulk effects.

The comparison of cantilever bending and lattice parameter changes suffers from potentially different strains. Cantilever bending setups typically use a very stiff glass substrate, whereas the samples of the measurements shown here, were deposited on a rather soft polyimide substrate, which might allow a certain stress relaxation. As a consequence, the apparent surface stress, and therefore the change in lattice parameter could be higher on a stiff substrate. In the most extreme estimation (i.e. the polyimide substrate does not contribute to the substrate stiffness), $f^{\text {app }}\left(\epsilon_{\|}^{t}=0\right)$ and $f^{\text {app }}\left(\epsilon_{\|}^{t}\right)$ differ by a factor of 3 , as will be discussed in more detail in the following section. There are some indications for certain stress relaxation mechanisms, especially the measured drift which is clearly visible in Figure 6.

The Interrelation of the lattice parameter and the potential-straincoupling parameter. Equation 4 links real and apparent surface 
Table I. Parameters of the sample layers.

\begin{tabular}{lccc} 
Material & $\begin{array}{c}\text { Thickness } \\
(\mathrm{nm})\end{array}$ & $\begin{array}{c}\text { Elastic } \\
\text { modulus } \\
(\mathrm{GPa})\end{array}$ & $\begin{array}{c}\text { Poisson's } \\
\text { ratio }\end{array}$ \\
\hline Polyimide $^{\mathrm{a}, \mathrm{b}}$ & 75000 & 2.5 & 0.34 \\
Polyimide $^{\mathrm{c}}$ & 75000 & 0 & 0.34 \\
Titanium $^{31}$ & 20 & 120.2 & 0.361 \\
Gold $^{31}$ & 55 & 78.5 & 0.42 \\
& & & \\
afast & & \\
baccording to the manufacturer & & \\
c slow & &
\end{tabular}

stress to the PSCP, as long as the results are independent on the different characteristic times.

$$
\varsigma=\frac{\mathrm{d}\left(f+f^{\text {app }}\right)}{\mathrm{d}\left(q+q^{\text {app }}\right)} \stackrel{\mathrm{d} f / \mathrm{d} q=0}{\approx} B_{\mathrm{Au}} h_{\mathrm{Au}} \frac{\mathrm{d} \epsilon_{\|}}{\mathrm{d}\left(q+q^{\text {app })}\right.}
$$

To interrelate the PSCP and the elastic strain, it is meaningful to multiply Equation 21 with the current density:

$$
\underbrace{\frac{\mathrm{d}\left(q+q^{\mathrm{app}}\right)}{\mathrm{d} t}}_{=I / A} \stackrel{\mathrm{d} f / \mathrm{d} q=0}{\approx} B_{\mathrm{Au}} h_{\mathrm{Au}} \underbrace{\frac{\mathrm{d} \epsilon_{\|}^{e}}{\mathrm{~d}\left(q+q^{\mathrm{app}}\right)} \frac{\mathrm{d}\left(q+q^{\mathrm{app}}\right)}{\mathrm{d} t}}_{=\mathrm{d} \epsilon_{\|}^{e} / \mathrm{d} t}
$$

The absence of real surface stress changes (besides UPD/UPDs) is indicated by the correlation of elastic strain and cantilever results (see Figure 8 ). The stiffness of the substrate on the other hand cannot be treated independent of the characteristic time. It was observed, that the polyimide substrate lost its tension after a few days, which indicates creep relaxation. This might be linked to uptake and redistribution of solvents in the polyimide substrate, which can reduce stresses. ${ }^{32}$ Hence, it has to be assumed, that the stiffness contribution of the polyimide strongly depends on the characteristic time.

For fast strain variations as applied during DECMA measurements, the polyimide will behave stiff, whereas for slow strain variations during cyclic voltammetry, it will behave soft. Using Equation 15 and parameters, as displayed in Table I, to calculate $\epsilon_{\|}^{e}$ (fast) : $\epsilon_{\|}^{e}$ (slow) leads to a ratio of $3: 1$.

The comparison in Equation 22 is then plotted in Figure 9. A good qualitative correlation except for UPD/UPDs and possible electrolyte decomposition can be observed. Quantitative differences (here by a factor of 3), which are visible by the different scale bars of the ordinates, most probably have their origin in the above described stress relaxation processes.

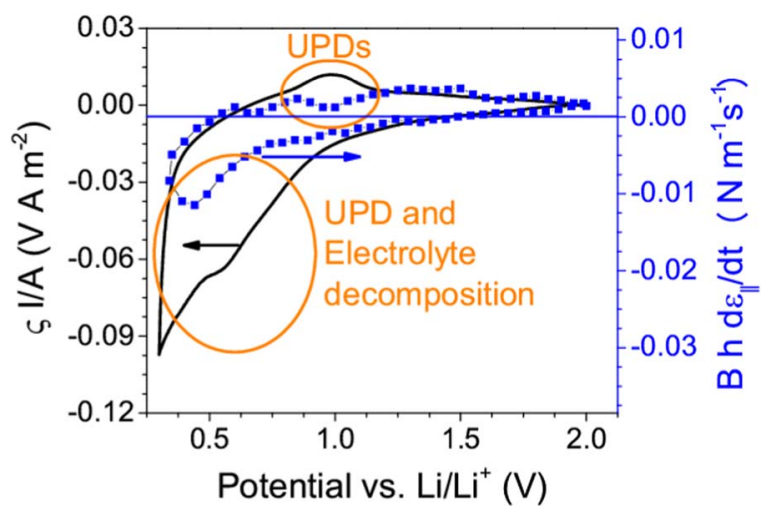

Figure 9. Qualitative comparison of the left hand side (black line) and the right hand side (blue symbols) of Equation 22. The obvious proportionality of both curves is only broken where well known surface stress changes and suspected slow processes (electrolyte decomposition) appear. Please note the different scaling of the two y-axes and the offset.

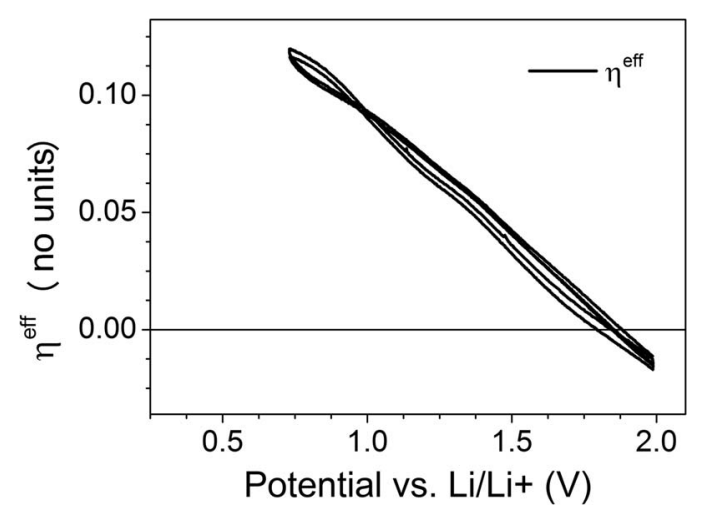

Figure 10. Effective in-plane strain-concentration coefficient $\eta^{\text {eff }}$ of an $\mathrm{Au}$ film in an Li electrolyte as a function of the applied potential.

Microscopic mechanisms. - Our results indicate the existence of a bulk mechanism which is able to reversibly induce a strain characteristic for a planar stress state on a thin film electrode in contact with a substrate. A planar stress state which changes electrochemically, can only be the result of a stress-free strain, which is counteracted by the substrate. In a substitutional alloy, a lattice parameter independent stress-free strain can only be accomplished by the creation of new inplane lattice places. The lattice of a solid can only be altered at grain boundaries, dislocations, and surfaces ${ }^{10}$ Hence, lattice places need to be created/removed at grain boundaries or dislocations to change the apparent surface stress. It should be emphasized here, that processes which have a negligible impact on the stress-free strain and the lattice parameter, like diffusion, might appear additionally to the addition and removal of lithium to/from defects.

In case the PSCP is only correlated to bulk mechanisms, Equation 10 can be used to calculate the effective increase in volume with respect to the transferred charge $\eta_{\|}^{\text {eff }}=\eta q^{\text {app }} /\left(q+q^{\text {app }}\right)$ using materials parameter of gold (see Table I). The results presented in this work indicate, that for $\mathrm{CV}$-scans in the potential range of $2 \mathrm{~V}$ to $0.7 \mathrm{~V}$ vs. $\mathrm{Li} / \mathrm{Li}^{+}$, surface stress changes are negligible. Hence the PSCP can be used to calculate an effective $\eta^{\text {eff }}$ as shown in Figure 10.

The volume required for creating a substitutional lattice place can be calculated from gold-lithium alloy lattice parameter data ${ }^{21}$ to be $99 \%$ of the volume of a gold lattice place in the solid solution regime, while the lattice structure is conserved. This corresponds to a $\eta$ value of 0.33 , which is much higher than the highest value in Figure 10 of 0.12 . For grain boundary alloying the situation is different, because grain boundaries are less ordered. Hence lower $\eta_{\|}^{\text {eff }}$ can be expected, which fits our results. The slightly negative strain-concentration coefficient measured above $1.9 \mathrm{~V}$ vs. $\mathrm{Li} / \mathrm{Li}^{+}$indicates, that the width of the grain boundary can even be reduced in the beginning of lithiation. A second argument in favor of grain boundary alloying is the tendency, that with increasing film thickness, and therefore decreasing number of grain boundaries per volume (because of increasing grain size $\mathrm{e}^{35}$ ), the current density measured during cyclic voltammetry decreases $\left(10.3 \rightarrow 8.7 \rightarrow 6.8 \rightarrow 6.2 \mathrm{~A} / \mathrm{m}^{2}\right.$ current density for $30 \rightarrow 50 \rightarrow 80 \rightarrow 120 \mathrm{~nm}$ thick films, respectively, measured during cyclic voltammetry (2 to $0.3 \mathrm{~V}$ ) with a scan rate of $10 \mathrm{mV} / \mathrm{s}$ at $1.3 \mathrm{~V}$ vs. $\left.\mathrm{Li} / \mathrm{Li}^{+}\right)$. The results strongly indicate, that $\eta^{\text {eff }}$ is correlated to grain boundary alloying and has a strong dependency on the electrode potential. Such a strong dependency might have an important impact on processes like storage, ${ }^{33}$ trapping $^{34}$ and diffusion of lithium in grain boundaries.

\section{Conclusions}

A customized DECMA stage suitable for operation inside a glove box was designed. Additionally, a possibility for in-situ measurement of the substrate strain has been introduced. 
In Combined surface and bulk PSCP section it was shown, that the PSCP of a system with two different contributions is the effectivecapacity weighted average of both contributions. An expansion to more contributions can be done in a similar way. In this study, a thin gold film was used as a model electrode to demonstrate that it is possible to measure the PSCP of a lithium electrode. The results revealed that this parameter is strongly dependent on the electrode potential. It changes from $\approx-0.4 \mathrm{~V}$ at a potential of $2 \mathrm{~V} \mathrm{vs.} \mathrm{Li}^{\prime} / \mathrm{Li}^{+}$to $2.1 \mathrm{~V}$ at a potential of $0.55 \mathrm{~V} \mathrm{vs}$. $\mathrm{Li} / \mathrm{Li}^{+}$. To our knowledge such strong dependency of the electro-chemo-mechanical coupling has neither been considered nor measured so far for lithium alloying.

The results were used to calculate an interval for the surface stress changes due to underpotential deposition of $[-3.1 ;-1.95 \mathrm{~N} / \mathrm{m}]$ which fits to literature results calculated using density functional theory of $-2.8 \mathrm{~N} / \mathrm{m}^{16}$

A setup for orientation dependent, in-situ X-ray diffraction was introduced. It was used to measure the electrochemically induced change in lattice parameter which was found to vary between $0.002 \%$ and $-0.054 \%$ in-plane and between $-0.003 \%$ and $0.089 \%$ out-ofplane, with a relation characteristic for a planar stress state.

The combination of both measurements showed a good qualitative agreement. Moreover, it offered the unique possibility to separate surface and bulk stresses from in-situ measurements. It could be shown, that for this model electrode, the apparent surface stress due to bulk phenomena outweighs the actual surface stress due to underpotential deposition. This demonstrates the importance of considering bulk contributions when measuring surface stress changes. Detailed analysis of the results lead us to the conclusion, that the dominating mechanism responsible for bulk stresses is lithium entering grain boundaries.

We suspect, that the strong dependency of the strain concentration coefficient on the electrode potential has a strong impact on storage, ${ }^{33}$ trapping ${ }^{34}$ and diffusion of lithium in grain boundaries.

\section{Acknowledgments}

We acknowledge the Helmholtz-Zentrum Berlin für Materialien und Energie $\mathrm{GmbH}$ (HZB) for provision of synchrotron radiation beamtime at beamline KMC-2 of BESSY II.

\section{References}

1. J. Christensen, P. Albertus, R. S. Sanchez-Carrera, T. Lohmann, B. Kozinsky, R. Liedtke, J. Ahmed, and A. Kojic, J. Electrochem. Soc., 159(2), R1 (2012).
2. B. Scrosati, J. Hassoun, and Y.-K. Sun, Energy Environ. Sci., 4(9), 3287 (2011).

3. W. J. Zhang, J. Power Sources, 196(1), 13 (2011).

4. R. A. Huggins, J. Power Sources, 81-82, 13 (1999).

5. D. Fauteux and R. Koksbang, J. Appl. Electrochem., 23(1), 1 (1993).

6. A. Mukhopadhyay and B. W. Sheldon, Prog. Mater. Sci., 63, 58 (2014)

7. L. Ji, Z. Lin, M. Alcoutlabi, and X. Zhang, Energy Environ. Sci., 4(8), 2682 (2011).

8. S. Goriparti, E. Miele, F. De Angelis, E. Di Fabrizio, R. Proietti Zaccaria, and C. Capiglia, J. Power Sources, 257, 421 (2014).

9. F. Hao, X. Gao, and D. Fang, J. Appl. Phys., 112(10) (2012).

10. F. Larché and J. W. Cahn, Acta Metall., 21, 1051 (1973).

11. Y. F. Gao and M. Zhou, J. Power Sources, 230, 176 (2013).

12. V. A. Sethuraman, V. Srinivasan, A. F. Bower, and P. R. Guduru, J. Electrochem. Soc., 157(11), A1253 (2010).

13. T. Ichitsubo, S. Yukitani, K. Hirai, S. Yagi, T. Uda, and E. Matsubara, J. Mater. Chem., 21(8), 2701 (2011).

14. T. Ichitsubo, S. Yagi, T. Doi, S. Yukitani, K. Hirai, and E. Matsubara, J. Electrochem. Soc., 159(1), A14 (2012)

15. S. P. V. Nadimpalli, V. A. Sethuraman, G. Bucci, V. Srinivasan, A. F. Bower, and P. R. Guduru, J. Electrochem. Soc., 160(10), A1885 (2013).

16. H. Tavassol, M. K. Y. Chan, M. G. Catarello, J. Greeley, D. G. Cahill, and A. A. Gewirth, J. Electrochem. Soc., 160(6), A888 (2013).

17. M. Smetanin, Q. Deng, and J. Weissmüller, Phys. Chem. Chem. Phys., 13(38), 17313 (2011).

18. J. Weissmüller, In Electrocatalysis, Vol. 14, eds R. C. Alkire, D. M. Kolb, L. A. Kibler, and J. Lipkowski, Wiley-VCH, Germany, (2013).

19. R. Shuttleworth, Proc. Phys. Soc. Sect. A, 63(5), 444 (2002)

20. R. N. Viswanath and J. Weissmüller, Acta Mater, 61(16), 6301 (2013).

21. G. Kienast, J. Verma, and W. Klemm, Zeitschrift für Anorg. und Allg. Chemie, 310(3), 143 (1961).

22. L. Martin, H. Martinez, D. Poinot, B. Pecquenard, and F. Le Cras, J. Power Sources, 248, 861 (2014)

23. ,,B. Philippe, A. Mahmoud, J. B. Ledeuil, M. T. Sougrati, K. Edström, R. Dedryvère, D. Gonbeau, and P. E. Lippens, Electrochim. Acta, 123, 72 (2014).

24. K. Tasaki, A. Goldberg, J.-J. Lian, M. Walker, A. Timmons, and S. J. Harris, J. Electrochem. Soc., 156(12), A1019 (2009).

25. Q. Deng, Dynamic Electro-Chemo-Mechanical Analysis at the Metal-Electrolyte Interface. Phd. thesis, TUHH, (2014)

26. C. Bauer and R. Farris, Polym. Eng. Sci., 14650(8), 1107 (1989),

27. A. Erko, I. Packe, C. Hellwig, M. Fieber-Erdmann, O. Pawlizki, M. Veldkamp, and W. Gudat, In AIP Conf. Proc., P. Pianetta, J. Arthur, and S. Brennan, editor, volume 521 of AIP Conference Proceedings, 415-418 (Amer Inst Physics, 2 Huntington Quadrangle, Ste 1NO1, Melville, NY 11747-4501 USA, 2000).

28. F. Sánchez-Bajo and F. L. Cumbrera, J. Appl. Crystallogr., 30(5), 550 (1997).

29. D. Aurbach, M. Daroux, P. Faguy, and E. Yeager, J. Electroanal. Chem. Interfacial Electrochem., 297(1), 225 (1991).

30. M. Smetanin, R. N. Viswanath, D. Kramer, D. Beckmann, T. Koch, L. A. Kibler, D. M. Kolb, and J. Weissmüller, Langmuir, 24(16), 8561 (2008).

31. William Francis Gale, Terry C. Totemeier, C. J. S., Elastic properties, damping capacity and shape memory alloys. Elsevier, (2004).

32. B. D. Harper, J. M. Rao, V. H. Kenner, and C. H. Popelar, J. Electron. Mater, 26(7), 798 (1997).

33. L. Y. Beaulieu, D. Larcher, R. A. Dunlap, and J. R. Dahn (2000)

34. H. Li, L. Shi, Q. Wang, L. Chen, and X. Huang, Solid State Ionics, 148(3-4), 247 (2002).

35. C. V. Thompson, Annu. Rev. Mater. Sci., 20(1), 245 (1990). 